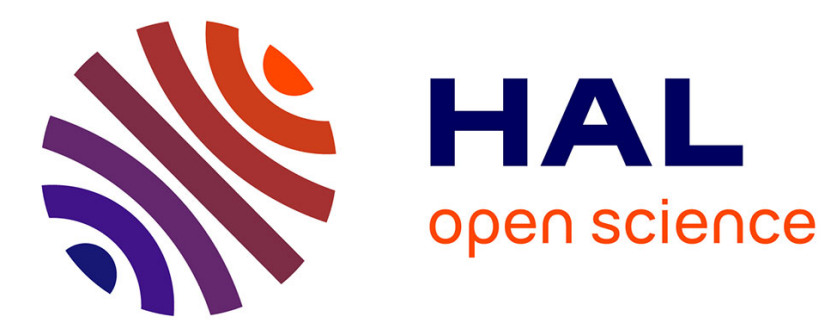

\title{
Low temperature low frequency noise in oxide and reoxidized-nitrided oxide films
}

\author{
R. Divakaruni, R. Peterson, S. Nystrom, C. Viswanathan
}

\section{To cite this version:}

R. Divakaruni, R. Peterson, S. Nystrom, C. Viswanathan. Low temperature low frequency noise in oxide and reoxidized-nitrided oxide films. Journal de Physique IV Proceedings, 1994, 04 (C6), pp.C6-43-C6-48. 10.1051/jp4:1994607 . jpa-00253101

\section{HAL Id: jpa-00253101 https://hal.science/jpa-00253101}

Submitted on 1 Jan 1994

HAL is a multi-disciplinary open access archive for the deposit and dissemination of scientific research documents, whether they are published or not. The documents may come from teaching and research institutions in France or abroad, or from public or private research centers.
L'archive ouverte pluridisciplinaire HAL, est destinée au dépôt et à la diffusion de documents scientifiques de niveau recherche, publiés ou non, émanant des établissements d'enseignement et de recherche français ou étrangers, des laboratoires publics ou privés. 


\title{
Low temperature low frequency noise in oxide and reoxidized-nitrided oxide films
}

\author{
R. Divakaruni, R. Peterson*, S. Nystrom* and C.R. Viswanathan \\ Electrical Engineering Department, University of California, Los Angeles, California, 90024, U.S.A. \\ * Hughes Aircraft Company, El Segundo, California, U.S.A.
}

\begin{abstract}
The reliability of oxide and reoxidized-nitrided oxide (RNO) gate dielectrics was examined by comparing the low frequency noise characteristics at low temperature of p-channel devices before and after positive F-N stress. The RNO devices were found to have a better noise performance after F-N stress and were also found to have less interface state generation due to the warm-up process. However, the creation of negative fixed charge in RNO devices has a severe effect on the threshold voltage.
\end{abstract}

\section{INTRODUCTION}

Even though low frequency noise has been studied extensively in the strong inversion regime, very few studies have been conducted in the weak inversion and subthreshold regions of operation of the MOS device. The subthreshold region of operation is particularly important at cryogenic temperatures for focal plane array applications and noise minimization is of extreme importance in such applications [1]. Recently, oxynitride gate dielectrics have been suggested for use at cryogenic temperatures [2]. In this paper, we compare oxide and Reoxidized-Nitrided Oxide (RNO) samples by measuring the low frequency noise before and after positive F-N stress on p-channel devices. Since the subthreshold region of operation is particularly important at cryogenic temperatures for focal plane array applications [3], we measured the noise in the weak inversion regime.

\section{EXPERIMENTAL}

The transistors had $W / L$ equal to $100 / 10 \mu m$ and were fabricated in a p-well process with a phosphorus substrate doping of $10^{15} \mathrm{~cm}^{-3}$. The thermal oxide samples were bulk non-LDD nMOS transistors with oxide thickness of $180 \AA$. The RNO samples were prepared in the following manner. $120 \AA$ oxide was first thermally grown to form the gate oxide. Rapid thermal processing (RTP) in $\mathrm{NH}_{3}$ ambient was then used to obtain nitridation of the oxide. Finally, the reoxidized nitrided oxide was obtained by RTP in dry $\mathrm{O}_{2}$ ambient. The samples were bonded on to a chip carrier which was mounted on the cold tip of a helium dewar. The transconductance $\left(g_{m}\right)$ was measured 
with a HP 4142 system controlled by a personal computer. The low frequency noise was measured in an automated system at a drain voltage of $100 \mathrm{mV}$ for different drain currents. The noise was measured with a HP3561A dynamic signal analyzer and a custom made amplifier [4] which consists of three stages. The first stage is a transimpedance amplifier (current to voltage converter) while the second and third stages are $10 \times$ voltage amplifiers. The noise was measured for the virgin device at room and low temperatures. The device was then stressed at $10 \mathrm{~K}$ with a current density of $0.1 \mathrm{~m} \mathrm{Am}^{-2}$ for $20 \mathrm{sec}$ and the noise measured at the same temperatures as earlier. The device was next warmed to room temperature and then cooled to $10 \mathrm{~K}$ where successive $\mathrm{F}-\mathrm{N}$ stresses of $0.2 \mathrm{~mA} \mathrm{~cm}^{-2}, 0.5 \mathrm{~mA} \mathrm{~cm} \mathrm{~cm}^{-2}$ and $1 \mathrm{~mA} \mathrm{~cm}^{-2}$ for 20 seconds each were performed. After the noise was measured at $10 \mathrm{~K}$, the device warmed to room temperature. After a few days, the device was cooled down again to $10 \mathrm{~K}$ and the noise measurement repeated from $10 \mathrm{~K}$ through $40 \mathrm{~K}$ to study the change in interface states due to thermal cycling [5].

\section{RESULTS ANE DISCUSSION}

Figure 1 shows the drain current versus the gate voltage (Id- $\mathrm{Vg}$ ) and the transconductance $\left(g_{m}\right)$ curves for the RNO device before and after the $0.1 \mathrm{~mA} \mathrm{~cm} \mathrm{~cm}^{-2} \mathrm{~F}-\mathrm{N}$ stress (1st F-N stress) at $10.5 \mathrm{~K}$. We see that the threshold voltage becomes less negative (which is indicative of the creation of a negative fixed charge) and there is no degradation in the measured $g_{m}$. This implies that the electrons injected from the cathode get trapped in the RNO oxide causing a negative charge buildup. These charges are also not trapped close to the silicon interface as this would cause some degradation in the transconductance which is not observed. Figure 2 shows the Id- $\mathrm{Vg}$ and $g_{m}$ curves for the oxide device under similar F-N stress conditions. We see that the threshold voltage in this case becomes more negative suggesting a positive charge buildup in the oxide while the degradation of the peak measured $g_{m}$ is due to the generation of interface states. The positive charge buildup in the oxide is due to impact ionization caused by the hot electrons near the anode [6]. The impact ionization near the anode causes electrons and holes. The holes move toward the cathode and some are trapped in the process. This causes the positive charge buildup. Some of the injected electrons also recombine with the trapped holes near the cathode causing interface states [6].

Figure 3 shows the low frequency noise measured before and after the $0.1 \mathrm{~mA} \mathrm{~cm}^{-2}$ positive F-N stress on the RNO sample at $10 \mathrm{~K}$. We see that the low frequency noise has remained unchanged in the weak inversion regime. In our observation, the noise in weak inversion did not change appreciably with drain current making it possible to compare noise at different drain current levels. Figure 4 shows the low frequency noise measured before and after the $0.1 \mathrm{~mA} \mathrm{~cm} \mathrm{~cm}^{-2}$ positive F-N stress on the oxide sample at $10 \mathrm{~K}$. We observe an increase due to $\mathrm{F}-\mathrm{N}$ stress in the low frequency noise in weak inversion. These results correlate with the transconductance degradation in the two samples.

The cooled device was next subject to consecutive F-N stress. We noticed a change in the $g_{m}$ and Id- $\mathrm{Vg}$ curves after the consecutive positive $\mathrm{F}-\mathrm{N}$ stresses of $0.2 \mathrm{~mA} \mathrm{~cm}^{-2}, 0.5 \mathrm{~mA} \mathrm{~cm}^{-2}$ and $1 \mathrm{~mA} \mathrm{~cm} \mathrm{~cm}^{-2}$ for 20 seconds each. Figure 5 shows the $g_{m}$ and $\mathrm{Id}=\mathrm{Vg}$ curves for the RNO device before the $0.2 \mathrm{~mA} \mathrm{~cm}^{-2}$ stress (2nd F-N stress) and after the $1 \mathrm{~mA} \mathrm{~cm}^{-2}$ stress (4th F-N stress). The threshold voltage has become significantly less negative indicative of a large negative fixed charge but the peak $g_{m}$ value has still not changed. A similar set of curves for the oxide device (before the $0.2 \mathrm{~mA} \mathrm{~cm}^{-2}$ and after the $1 \mathrm{~mA} \mathrm{~cm}^{-2}$ ) is shown in Figure 6. We see that the transconductance has degraded significantly after the consecutive stressing and there is a significant amount of positive fixed charge built up in the oxide causing the threshold voltage to become significantly more negative.

When the noise of the RNO device after the consecutive F-N stress is compared with the noise of the virgin RNO sample at about $10 \mathrm{~K}$ as shown in Figure 7, we see that the noise increases over the entire spectrum. The data indicates that the RNO samples degrade at higher levels of F-N stress as compared to the oxide samples which showed an increased noise even after a positive F-N 
stress of $0.1 \mathrm{~m}^{\prime} \mathrm{cm}^{-2}$ for 20 seconds. On comparison with the virgin device, the noise of the oxide sample after the consecutive F-N stresses increases significantly as shown in Figure 8. There is a much larger increase in the noise of the oxide samples after the consecutive F-N stress than in the RNO samples after the consecutive stress.

The device was warmed to room temperature after the consecutive F-N stress and then cooled again after a few days. We then measured the Id- $\mathrm{Vg}$ as well as the $g_{m}$ on both the RNO and oxide devices. Figure 9 shows that there is significant relaxation of the threshold voltage (which becomes more negative) for the RNO device due to the warmup process, but there is no change in the $g_{m}$ (transconductance) peak. This indicates the rearrangement of the negative fixed charge and the absence of the formation of interface states. For the oxide device, however, we see that there is little change in the threshold voltage (not much change in the fixed charge in the oxide), but the transconductance has severely degraded (Figure 10) indicating the formation of a significant number of interface states in the oxide sample due to the warmup process. This increase in interface states in the oxide sample due to the warming up and subsequent cooling of the device should affect the measured low frequency noise.

For the RNO sample, we see that there is a small reduction in the noise due to the warmup process as shown in Figure 11. indicating that there has been some annealing of the interface states due to the warmup process after the consecutive F-N stress. In general, we found that the noise of the RNO device either decreased or remained the same due to the warmup process. The threshold voltage, however, showed a significant relaxation due to the warmup process. For the oxide sample, we see that there is a significant increase in the noise due to the warmup process over the entire frequency spectrum as shown in Figure 12. The figure clearly demonstrates a significant increase in interface states in the oxide sample due to the warmup process after the consecutive F-N stress. In general, we found that the noise of the oxide device always increased due to the warmup process indicating the generation of interface states.

\section{CONCLUSION}

In conclusion, the RNO samples have better noise characteristics under F-N stress and show no interface state generation on warming up to room temperature. We found negative charge trapping in the RNO samples due to the F-N stress and positive charge creation in the oxide samples due to the F-N stress. There is a larger shift in threshold voltage due to fixed charge creation in the RNO samples.

\section{REFERENCES}

[1] D. H. Seib, "Cryogenic Electronics for Infrared Focal Plane Arrays", Proc. Symp. Low Temp. Electronics \& High Temp. Superconductors, pp.499-517,1988

[2]R. M. Glidden et al, "Optimization of Cryogenic CMOS processes for Sub-10K Applications", SPIE - Infrared Readout Electronics vol. 1684, 1992.

[3] C. Schutte and P. Rademeyer, "Subthreshold 1/f Noise Measurements in MOS Transistors Aimed at Optimizing Focal Plane Array Signal Processing", Analog Int. Cir. and Sig. Proc. Vol. 2, pp. 171-177, 1992.

[4] Jimmin Chang, "Flicker Noise in Enhancement, Degraded and Depletion MOSFET's", UCLA M.S. Thesis 1988.

[5] J. A. Lopez-Villanueva et al, "Analysis of the effects of constant-current Fowler-Nordheimtunneling injection with charge trapping inside the potential barrier", Journal of Applied Physics, Vol. 70, pp. 3712, 1991.

[6] D. J. DiMaria, E. Cartier and D. Arnold, "Impact Ionization, Trap Creation, Degradation, and Breakdown in Silicon Dioxide Films on Silicon", Journal of Applied Physics, Vol. 73 (7), 1 April, 1993. 


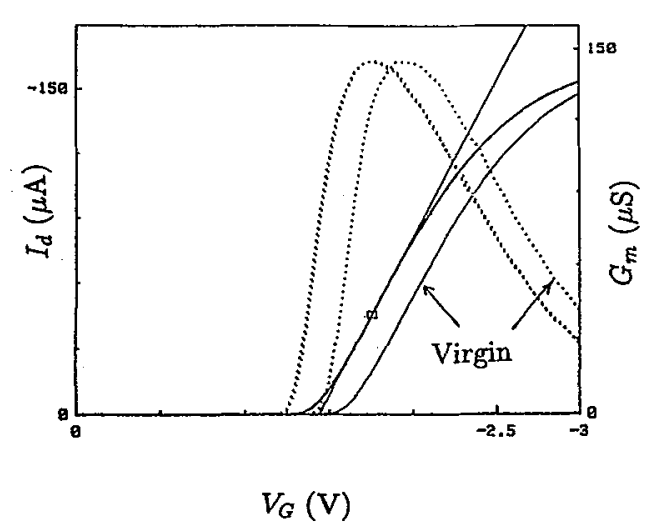

Fig. $1 I_{d}-V_{g}$ and $G_{m}$ curves for the virgin pchannel device with a RNO dielectric and after the $0.1 \mathrm{~mA} \mathrm{~cm}^{-2}$ positive $\mathrm{F}-\mathrm{N}$ stress (1st F-N stress).

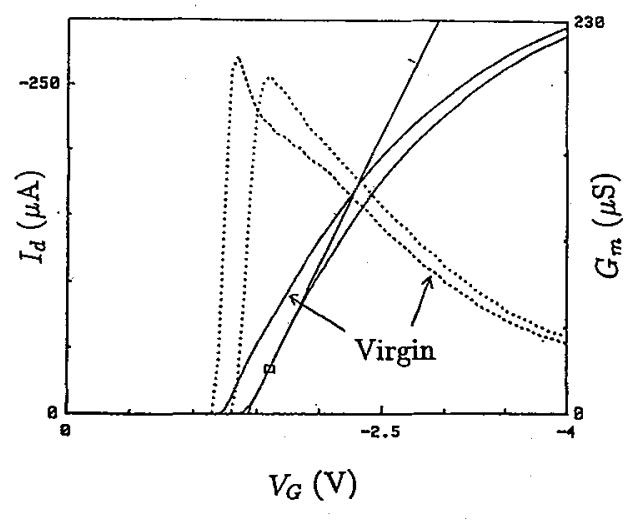

Fig. $2 I_{d}-V_{g}$ and $G_{m}$ curves for the virgin pchannel device with an oxide dielectric and after the $0.1 \mathrm{~mA} \mathrm{~cm}^{-2}$ positive F-N stress (1st $\mathrm{F}-\mathrm{N}$ stress).

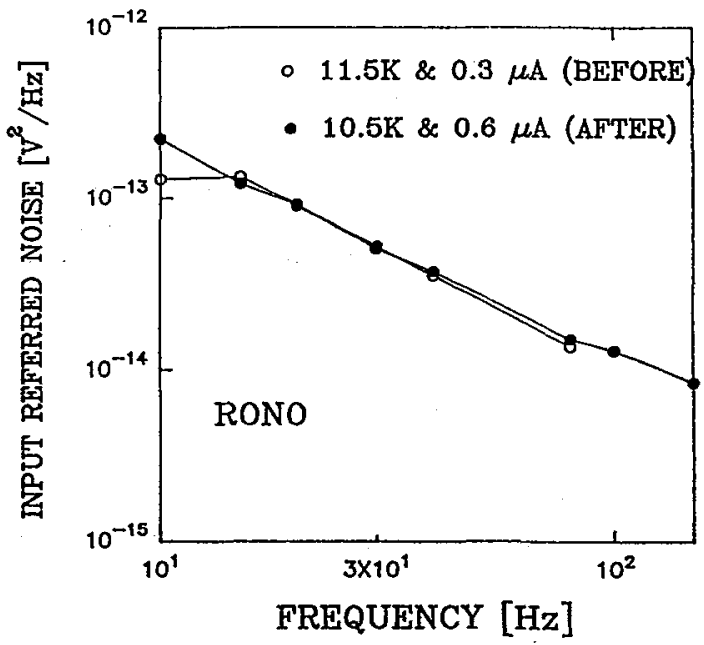

Fig. 3 Plot of the input referred noise spectrum for the RNO sample before stress and after the

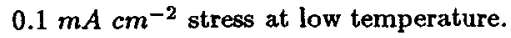

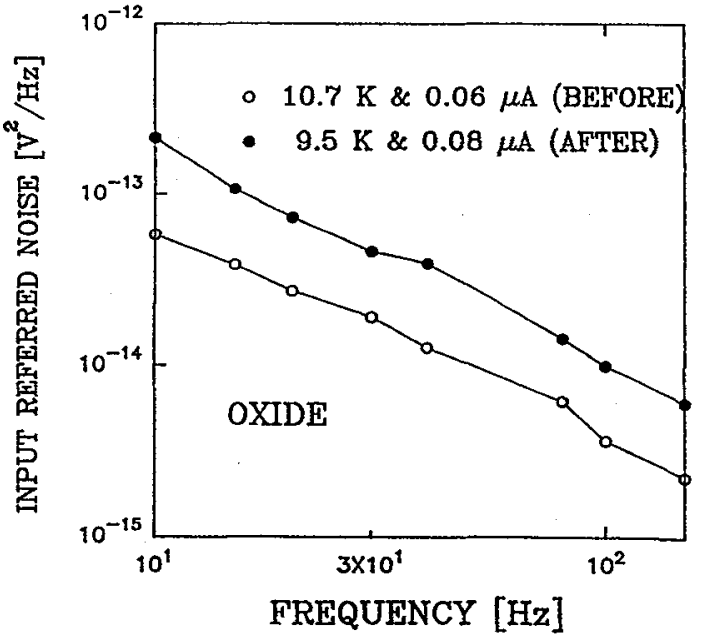

Fig. 4 Plot of the input referred noise spectrum for the oxide sample before stress and after the $0.1 \mathrm{~mA} \mathrm{~cm}^{-2}$ stress at low temperature. 


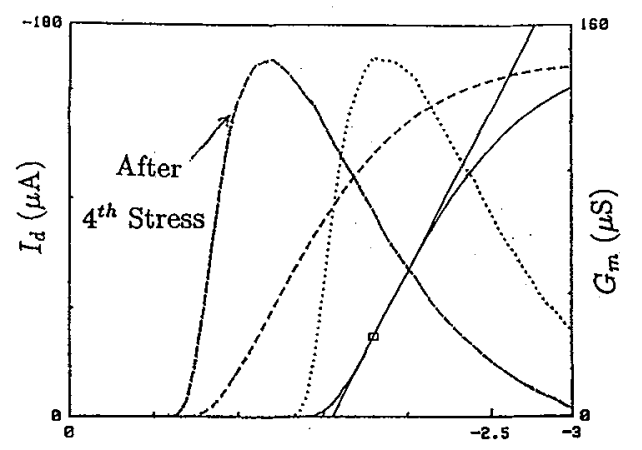

$V_{G}(\mathrm{~V})$

Fig. $5 I_{d}-V_{g}$ and $G_{m}$ curves for the p-channel device with a RNO dielectric before the $0.2 \mathrm{~mA} \mathrm{~cm}^{-2}$ positive F-N stress (2nd F-N stress) and after the $1 \mathrm{~mA} \mathrm{~cm}^{-2}$ positive F-N stress (4th F-N stress).

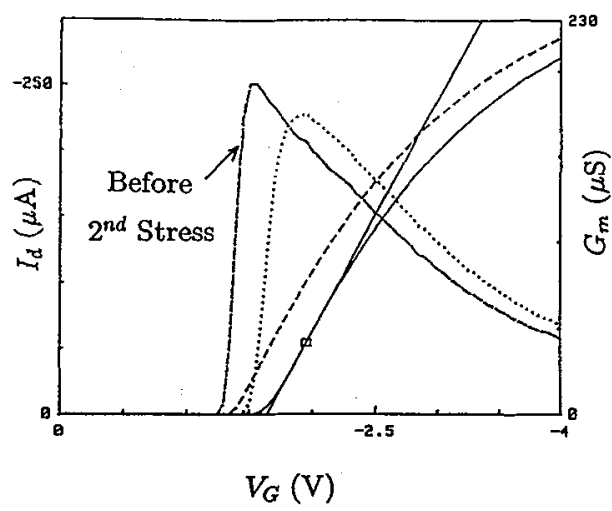

Fig. $6 I_{d}-V_{g}$ and $G_{m}$ curves for the p-channel device with an oxide dielectric before the $0.2 \mathrm{~mA} \mathrm{~cm}-2$ positive F-N stress (2nd F-N stress) and after the $1 \mathrm{~mA} \mathrm{~cm}^{-2}$ positive F-N stress (4th F-N stress).

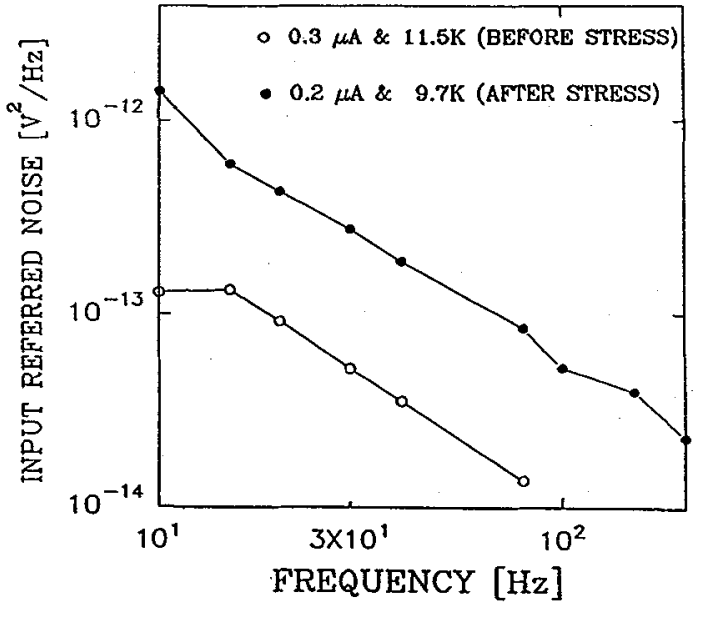

Fig. 7 Input referred noise of the virgin RNO device and after the 4th F-N stress at about 10K.

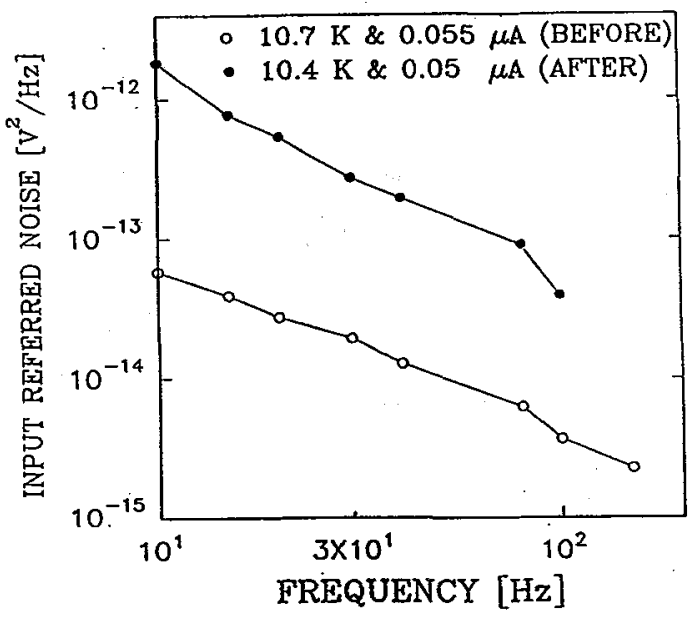

Fig. 8 Input referred noise of the virgin oxide device and after the 4th F-N stress at about 10K. 


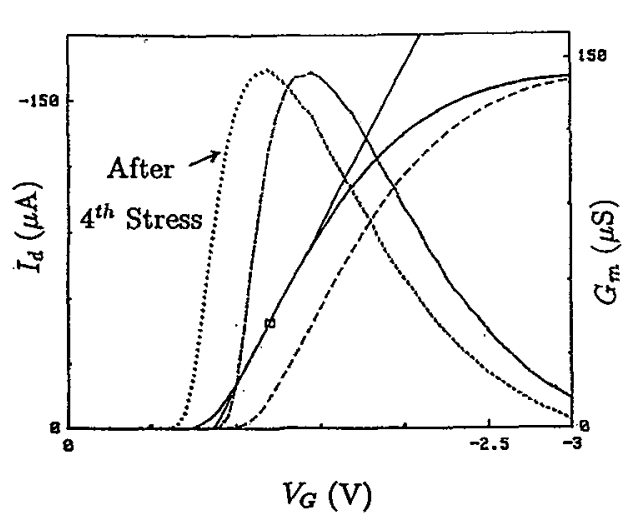

Fig. $9 I_{d}-V_{g}$ and $G_{m}$ curves for the p-channel device with a RNO dielectric after the 4th F-N stress and after the subsequent warmup and cool down (dashed lines).

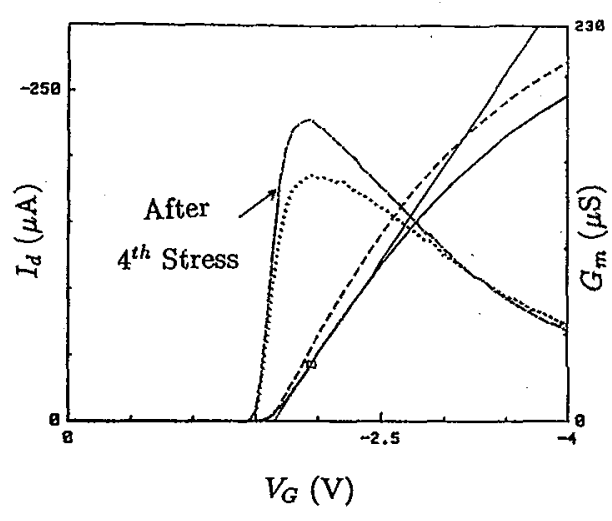

Fig. $10 I_{d}-V_{g}$ and $G_{m}$ curves for the p-channel device with an oxide dielectric after the 4 th $F-N$ stress and after the subsequent warmup and cool down (dot ted line $-G_{m}$ and solid line $-I_{d}-V_{g}$ ).

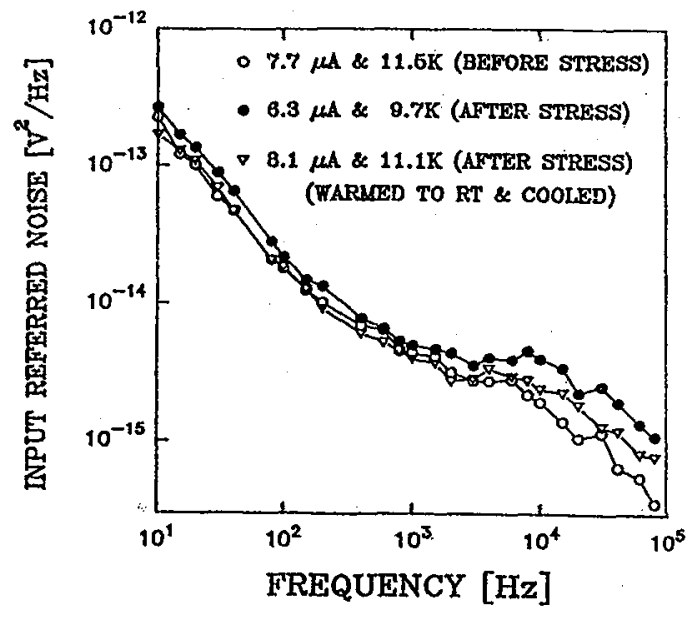

Fig. 11 Input referred noise of the virgin RNO device, after the 4 th $\mathrm{F}-\mathrm{N}$ stress and after the subsequent warmup and cooling at about $10 \mathrm{~K}$.

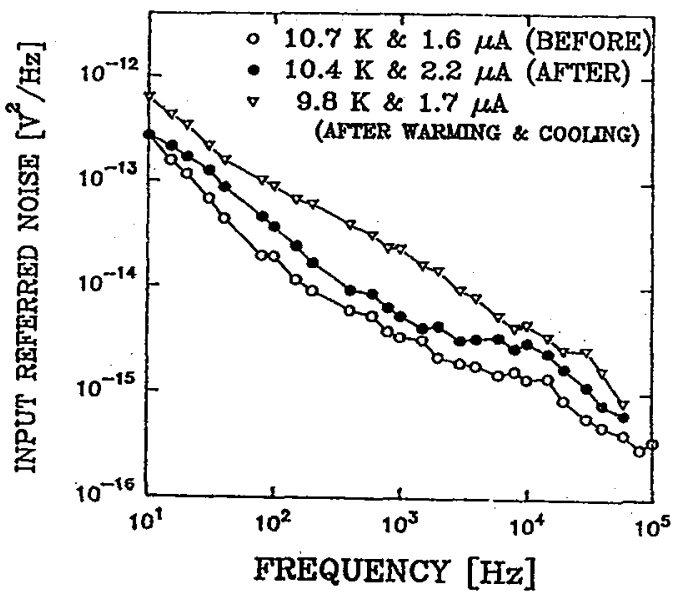

Fig. 12 Input referred noise of the virgin oxide device, after the 4th $\mathrm{F}-\mathrm{N}$ stress and after the subsequent warmup and cooling at about $10 \mathrm{~K}$. 